\title{
Global existence and blow-up of solutions for $p$-Laplacian evolution equation with nonlinear memory term and nonlocal boundary condition
}

\section{Zhong Bo Fang ${ }^{*}$ and Jianyun Zhang}

\section{"Correspondence:}

fangzb7777@hotmail.com

School of Mathematical Sciences,

Ocean University of China, Qingdao,

266100, P.R. China

\begin{abstract}
In this paper, we deal with an initial boundary value problem for a $p$-Laplacian evolution equation with nonlinear memory term and inner absorption term subject to a weighted linear nonlocal boundary condition. We find the effects of a weighted function as regards determining blow-up of nonnegative solutions or not and establish the precise blow-up estimate for the linear diffusion case under some suitable conditions.
\end{abstract}

Keywords: $p$-Laplacian evolution equation; nonlinear memory; global existence; blow-up; weight function

\section{Introduction}

In the past decades, there have been many works dealing with global existence and blowup properties of solutions for nonlinear parabolic equations, especially the initial boundary value problems with nonlocal terms in equations or boundary conditions, we refer to [1-10] and references therein. For the study of an initial boundary value problem for local parabolic equations with nonlocal boundary condition, we refer to [1-4]. For example, Friedman [1] studied the linear parabolic equation

$$
u_{t}-A u=0, \quad(x, t) \in \Omega \times(0, T),
$$

subject to the weighted linear nonlocal Dirichlet boundary condition

$$
u(x, t)=\int_{\Omega} f(x, y) u(y, t) d y, \quad(x, t) \in \partial \Omega \times(0, T)
$$

where $A$ is an elliptic operator,

$$
A=\sum_{i, j=1}^{n} a_{i, j}(x) \frac{\partial^{2}}{\partial x_{i} \partial x_{j}}+\sum_{i=1}^{n} b_{i}(x) \frac{\partial}{\partial x_{i}}+c(x) \quad \text { and } \quad c(x) \leq 0
$$

and the nonnegative continuous function $f(x, y)$ satisfies suitable conditions. He proved that when $\int_{\Omega} f(x, y) d y \leq \rho<1$, the solution approaches to 0 monotonously and exponen-

○2014 Fang and Zhang; licensee Springer. This is an Open Access article distributed under the terms of the Creative Commons Attribution License (http://creativecommons.org/licenses/by/2.0), which permits unrestricted use, distribution, and reproduction in any medium, provided the original work is properly cited. 
tially as $t \rightarrow \infty$. As regards more general discussions on an initial boundary value problem for a linear parabolic equation with a weighted linear nonlocal Neumann boundary condition, one can refer to [2] by Pao, where the following problem:

$$
\begin{aligned}
& u_{t}-L u=g(x, u), \quad x \in \Omega, t>0, \\
& B u=\int_{\Omega} f(x, y) u(y, t) d y, \quad x \in \partial \Omega, t>0, \\
& u(x, 0)=u_{0}(x), \quad x \in \Omega,
\end{aligned}
$$

where

$$
L u=\sum_{i, j=1}^{n} a_{i j}(x) u_{x_{i} x_{j}}+\sum_{j=1}^{n} b_{j}(x) u_{x_{j}}, \quad B u=\alpha_{0} \frac{\partial u}{\partial n}+u
$$

was considered. He studied the asymptotic behavior of solutions and found the influence of the weight function on the existence of global and blow-up solutions. Later, Akila [3] adopted the method of an upper-lower solution to consider the semilinear parabolic equation

$$
u_{t}=\Delta u+g(x, t, u), \quad(x, t) \in \Omega \times(0, \infty),
$$

under a similar weighted linear nonlocal boundary condition. Wang et al. [4] studied a porous medium equation with power form source term,

$$
u_{t}=\Delta u^{m}+u^{p}, \quad(x, t) \in \Omega \times(0,+\infty)
$$

under the weighted linear nonlocal Dirichlet boundary condition (1.1). By virtue of the method of an upper-lower solution, they obtained global existence, blow-up properties, and blow-up rate of solutions.

For the study of initial boundary value problem with nonlocal parabolic equation, especially the nonlocal problem with time-integral, we refer to [5-10]. Under a homogeneous Dirichlet boundary condition, $\mathrm{Li}$ and Xie [5] studied the nonlinear diffusion equation

$$
u_{t}-\Delta u=u^{q} \int_{0}^{t} u^{p} d s, \quad(x, t) \in \Omega \times(0, T)
$$

where $p \geq 0, q>0$. They obtained the sufficient conditions of global existence and blowup of solutions under appropriate critical conditions. Furthermore, under the following assumptions:

there exists $t_{0} \in(0, T)$ such that $u_{t}\left(x, t_{0}\right) \geq 0$ for all $x \in \bar{\Omega}$,

and

$$
\begin{aligned}
& \Omega=\left\{x \in R^{N}:|x|<R\right\}, \quad u_{0}(x)=u_{0}(|x|) \equiv u_{0}(r), \\
& u_{0}^{\prime}(r)<0 \quad \text { and } \quad u_{0}^{\prime \prime}(r)<0
\end{aligned}
$$


they derived the following blow-up rate:

$$
C_{1}(T-t)^{-\frac{2}{p-1}} \leq \max _{x \in \bar{\Omega}} u(x, t) \leq C_{2}(T-t)^{-\frac{2}{p-1}}, \quad t \rightarrow T,
$$

for the special case $p>1$ and $q=0$. It is necessary to point out that assumption (1.2) seems to be reasonable, but unfortunately, the authors of [5] did not give a relationship between $u_{0}(x)$ and equation (1.2). The characterization of the monotonicity condition (1.2) was given by Souplet in [6], who proved the existence of monotone in time solutions for the above problem and obtained the blow-up rate (1.4) without the assumption of condition (1.3).

Zhou et al. [7] considered the following singular diffusion equation with memory term:

$$
\left(u^{k}\right)_{t}-\Delta u=u^{q} \int_{0}^{t} u^{p} d s, \quad(x, t) \in \Omega \times(0, T),
$$

where $p \geq 0, q \geq 0,0<k<1$. They got similar results by the method of upper-lower solution. We should notice that this kind of equation can be turned into a degenerate porous medium equation by suitable transformation. In addition, for the system of porous medium equations with nonlinear memory terms and a homogeneous Dirichlet boundary condition, one can refer for example to $[8,9]$.

Recently, Liu and $\mathrm{Mu}[10]$ considered the following semilinear parabolic equation with memory term:

$$
u_{t}=\Delta u+u^{q} \int_{0}^{t} u^{p} d s, \quad(x, t) \in \Omega \times(0, T)
$$

subject to a weighted nonlinear nonlocal boundary,

$$
u(x, t)=\int_{\Omega} f(x, y) u^{l}(y, t) d y, \quad(x, t) \in \partial \Omega \times(0, T),
$$

where $p, q \geq 0$. They gave the conditions of global existence and blow-up of solutions and the blow-up rate of solutions for $q=0, l=1$ by establishing an auxiliary function.

In view of the works mentioned above, a nonlocal parabolic equation with time-integral term does not seem to be so much investigated as nonlocal equations with space-integral terms. Already at first glance, the problem with a memory term has some difficulties in proving the existence of non-global solutions. First if $t$ is sufficiently small, the nonlinear memory term vanishes, and then it is not clear whether the comparison principle holds in proving the existence of global small solutions. As far as we know, there are a few papers about the blow-up phenomenon for the $p$-Laplacian evolution equation with nonlinear memory term. Motivated by it, we consider the global existence and blow-up properties of the following $p$-Laplacian evolution equation with nonlinear memory term and inner absorption term:

$$
u_{t}-\operatorname{div}\left(|\nabla u|^{p-2} \nabla u\right)=u^{\alpha} \int_{0}^{t} u^{\beta} d s-k u^{m}, \quad(x, t) \in \Omega \times(0, T),
$$

subject to weighted linear nonlocal boundary and initial conditions

$$
u(x, t)=\int_{\Omega} f(x, y) u(y, t) d y, \quad(x, t) \in \partial \Omega \times(0, T),
$$




$$
u(x, 0)=u_{0}(x), \quad x \in \Omega
$$

where $p \geq 2, \alpha \geq 0, \beta>0, m>0, k>0$, and $\Omega \subset R^{N}(N \geq 1)$ is a bounded domain with smooth boundary. The weight function $f(x, y) \not \equiv 0$ in the boundary condition is continuous, nonnegative on $\partial \Omega \times \Omega$, and $\int_{\Omega} f(x, y) d y>0$ on $\partial \Omega$, while the nonnegative and nontrivial initial data $u_{0}(x) \in L^{\infty}(\Omega) \cap W_{0}^{1, p}(\Omega)$ satisfies the compatibility conditions $u_{t}(x, 0)=\operatorname{div}\left(\left|\nabla u_{0}(x)\right|^{p-2} \nabla u_{0}(x)\right)-k u_{0}^{s}(x)$ for $x \in \Omega$ and $u_{0}(x)=\int_{\Omega} f(x, y) u_{0}(y) d y$ for $x \in \partial \Omega$, which is the closed relationship for local solvability of our problem (1.5)-(1.7) (see Section 2).

The nonlocal diffusion model like equation (1.5) arises in many natural phenomena. In some sense, this kind of nonlocal problem is closer to the actual model than the local problem, such as the model of non-Newton flux through a porous medium, the model for compressible reactive gases, the model of population dynamics, and the model of biological species with human-controlled distribution (see [2, 11-14] and references therein). From a physics point of view, equation (1.5) with $p=2, m, \alpha, \beta=1$ and $k=0$ appears in the theory of nuclear reactor dynamics in which case the nonlocal term with time-integral is called the memory term [15]. In fact, there are some important phenomena formulated as parabolic equations which are coupled with weighted nonlocal boundary conditions in mathematical models, such as thermoelasticity theory. In this case, the solution $u(x, t)$ describes the entropy per volume of the material (see $[16,17])$.

Our main goal is to find the effects of weight function on global or non-global existence of solutions for problem (1.5)-(1.7), the suitable range of nonlinear exponent, and to give the blow-up rate estimate under some suitable conditions. In addition, we treat the nonlocal nonlinearity Hölder (non-Lipschitz) cases $m$ or $n \in(0,1)$, as well as the Lipschitz cases $m, n \geq 1$ in this paper. We get our main results by establishing a modified comparison principle, constructing the suitable upper and lower solutions (including the self-similar lower solutions, the eigenfunction argument and the technique of ordinary differential equation and so on) and the auxiliary function. Moreover, our results extend part of or all results in [8-10]. The detailed results are stated as follows.

- For arbitrary $f(x, y)>0$. If $\alpha+\beta>\max \{p-1, m\}$, then the solution of problem

(1.5)-(1.7) blows up in finite time for sufficiently large initial data.

- If $\int_{\Omega} f(x, y) \geq 1$, for $x \in \partial \Omega$. If $\alpha+\beta>m \geq 1$, then the solution of problem (1.5)-(1.7) blows up in finite time for all strictly positive initial dates with $T$ sufficiently large.

- If $\int_{\Omega} f(x, y)<1$, for $x \in \partial \Omega . \alpha+\beta>1, p=2$, the initial value $u_{0}(x)$ satisfies conditions $\left(\mathrm{H}_{1}\right)-\left(\mathrm{H}_{2}\right)$ (see Section 3$)$ and $u(x, t)$ is the blow-up solution of problem (1.2)-(1.4), then the blow-up rate is

$$
c(T-t)^{-\frac{1}{\alpha+\beta-1}} \leq u(x, t) \leq C(T-t)^{-\frac{1}{\alpha+\beta-1}}
$$

where $c=[(\alpha+\beta-1) T]^{-\frac{1}{\alpha+\beta-1}}, C=[(\alpha+\beta-1) \delta]^{-\frac{1}{\alpha+\beta-1}}$ and $\delta>0$ is a constant.

- If $\int_{\Omega} f(x, y)<1$, for $x \in \partial \Omega$,

(i) If $\alpha+\beta \leq \max \{p-1, m\}$, then the solution of problem (1.5)-(1.7) exists globally for small initial data.

(ii) If $p-1<\min \{\alpha, \beta+1, m\}$, and $\alpha+\beta>m$, then the solution of problem (1.5)-(1.7) exists globally for small initial data.

(iii) If $\alpha+\beta>m$, then the solution of problem (1.5)-(1.7) exists globally for small enough initial data. 
The rest of the paper is organized as follows. In Section 2, we give the preliminaries for our research. The proofs of blow-up results and blow-up rate of solutions are given in Section 3. In Section 4, we will deduce the results of global existence.

\section{Comparison principle and local existence}

Since equation (1.2) is degenerate when $p>2$, there is no classical solution in general. Hence, it is reasonable to find a weak solution. To this end, we first give the following definition of nonnegative weak solution of problem (1.5)-(1.7).

Definition 1 If the nonnegative function $u(x, t)$ satisfies the following conditions:

(1) $u \in C\left(0, T ; L^{\infty}(\Omega)\right) \cap L^{p}\left(0, T ; W_{0}^{1, p}(\Omega)\right)$,

$$
u_{t} \in L^{2}\left(0, T ; L^{2}(\Omega)\right), \quad u(x, 0)=u_{0}(x),
$$

(2) $\iint_{Q_{T}} u_{t} \phi d x d t+\iint_{Q_{T}}|\nabla u|^{p-2} \nabla u \cdot \nabla \phi d x d t$

$$
=\iint_{Q_{T}} \phi\left(u^{\alpha} \int_{0}^{t} u^{\beta} d s-k u^{m}\right) d x d t
$$

where $\phi \in L^{1}\left(0, T ; W^{1,2}(\Omega)\right) \cap L^{2}\left(Q_{T}\right)$ is nonnegative, $\left.\phi(x, t)\right|_{\partial \Omega}=0$, and $Q_{T}=\Omega \times(0, T)$.

$$
\text { (3) } u(x, t)=\int_{\Omega} f(x, y) u(y, t) d y, \quad x \in \partial \Omega \text {, }
$$

then $u(x, t)$ is called the weak solution of problem (1.5)-(1.7).

If the equalities in equations (2.1)-(2.3) are replaced by ' $\leq$ ' and ' $\geq$ ', we can get $\underline{u}(x, t)$ and $\bar{u}(x, t)$ which are called the lower solution and upper solution of problem (1.5)-(1.7), respectively.

The following modified comparison principle plays a crucial role in our proofs, which can be obtained by establishing a suitable test function and Gronwall's inequality.

Proposition 1 (Comparison principle) Suppose that $\underline{u}(x, t)$ and $\bar{u}(x, t)$ are the lower and upper solutions of problem (1.2)-(1.4), respectively. If $u(x, 0) \geq 0, \bar{u}(x, 0) \geq \varepsilon>0$ and $\underline{u}(x, 0) \leq \bar{u}(x, 0)$, where $\varepsilon$ is any positive constant, then $\underline{u}(x, t) \leq \bar{u}(x, t)$ in $\bar{\Omega} \times(0, T)$.

Proof For $x \in \Omega$, since $\underline{u}(x, t)$ and $\bar{u}(x, t)$ are the lower and upper solutions of problem (1.5)-(1.7), respectively, it follows that

$$
\begin{aligned}
& \iint_{Q_{T}}\left(\underline{u}_{t}-\bar{u}_{t}\right) \phi d x d t+\iint_{Q_{T}}\left(|\nabla \underline{u}|^{p-2} \nabla \underline{u}-|\nabla \bar{u}|^{p-2} \nabla \bar{u}\right) \cdot \nabla \phi d x d t \\
& \leq \iint_{Q_{T}} \phi\left[\underline{u}^{\alpha} \int_{0}^{t} \underline{u}^{\beta} d s-\bar{u}^{\alpha} \int_{0}^{t} \bar{u}^{\beta} d s-k\left(\underline{u}^{m}-\bar{u}^{m}\right)\right] d x d t \\
& =\iint_{Q_{T}} \phi\left[\left(\underline{u}^{\alpha}-\bar{u}^{\alpha}\right) \int_{0}^{t} \underline{u}^{\beta} d s+\bar{u}^{\alpha} \int_{0}^{t}\left(\underline{u}^{\beta}-\bar{u}^{\beta}\right) d s-k\left(\underline{u}^{m}-\bar{u}^{m}\right)\right] d x d t .
\end{aligned}
$$


Choose a test function $\phi=\chi_{[0, t]}(\underline{u}-\bar{u})_{+}=\max \{\underline{u}-\bar{u}, 0\}$ for $t>0$, where $\chi_{[0, t]}$ is a characteristic function defined on $[0, t]$, then we have

$$
\begin{aligned}
& \iint_{Q_{T}}(\underline{u}-\bar{u})_{t}(\underline{u}-\bar{u})_{+} d x d t+\iint_{Q_{T}}\left(|\nabla \underline{u}|^{p-2} \nabla \underline{u}-|\nabla \bar{u}|^{p-2} \nabla \bar{u}\right) \cdot \nabla(\underline{u}-\bar{u})_{+} d x d t \\
& \leq \iint_{Q_{T}}(\underline{u}-\bar{u})_{+}\left[\left(\underline{u}^{\alpha}-\bar{u}^{\alpha}\right) \int_{0}^{t} \underline{u}^{\beta} d s+\bar{u}^{\alpha} \int_{0}^{t}\left(\underline{u}^{\beta}-\bar{u}^{\beta}\right) d s-k\left(\underline{u}^{m}-\bar{u}^{m}\right)\right] d x d t \\
& \quad \leq \iint_{Q_{T}}\left[\Phi_{1}(x, t) \int_{0}^{t} \underline{u}^{\beta} d s+\bar{u}^{\alpha} T \Phi_{2}(x, t)+k \Phi_{3}(x, t)\right](\underline{u}-\bar{u})_{+}^{2} d x d t,
\end{aligned}
$$

where

$$
\begin{aligned}
& \Phi_{1}(x, t)=\alpha \int_{0}^{1}(\theta \underline{u}+(1-\theta) \bar{u})^{\alpha-1} d \theta, \\
& \Phi_{2}(x, t)=\beta \int_{0}^{1}(\theta \underline{u}+(1-\theta) \bar{u})^{\beta-1} d \theta, \\
& \Phi_{3}(x, t)=m \int_{0}^{1}(\theta \underline{u}+(1-\theta) \bar{u})^{m-1} d \theta .
\end{aligned}
$$

By Lemma 4.10 in [18], we know $\left(|\nabla \underline{u}|^{p-2} \nabla \underline{u}-|\nabla \bar{u}|^{p-2} \nabla \bar{u}\right) \cdot \nabla(\underline{u}-\bar{u})_{+} \geq 0$ for $p \geq 2$. Moreover, it follows from $m \geq 1, \alpha \geq 1, \beta \geq 1$ that $\Phi_{i}(i=1,2,3)$ is bounded, and if $0 \leq \alpha<1$, or $\beta<1$, we have $\Phi_{1} \leq \varepsilon^{\alpha-1}, \Phi_{2} \leq \varepsilon^{\beta-1}$ since $\underline{u}(x, 0) \geq 0, \bar{u}(x, 0) \geq \varepsilon>0$. Furthermore, because $\underline{u}(x, t)$ and $\bar{u}(x, t)$ are bounded functions, we can get

$$
\iint_{Q_{T}}(\underline{u}-\bar{u})_{t}(\underline{u}-\bar{u})_{+} d x d t \leq C \iint_{Q_{T}}(\underline{u}-\bar{u})_{+}^{2} d x d t .
$$

That is,

$$
\int_{\Omega}(\underline{u}-\bar{u})_{+}^{2} d x \leq \int_{\Omega}(\underline{u}(x, 0)-\bar{u}(x, 0))_{+}^{2} d x+C \iint_{Q_{T}}(\underline{u}-\bar{u})_{+}^{2} d x d t .
$$

Since $\underline{u}(x, 0) \leq \bar{u}(x, 0)$, it follows that

$$
\int_{\Omega}(\underline{u}-\bar{u})_{+}^{2} d x \leq C \iint_{Q_{T}}(\underline{u}-\bar{u})_{+}^{2} d x d t
$$

By Gronwall's inequality, we can deduce that $(\underline{u}-\bar{u})_{+}=0$, and so $\underline{u}(x, t) \leq \bar{u}(x, t)$ in $\Omega \times$ $(0, T)$.

For $x \in \partial \Omega, y \in \Omega$, we have

$$
\underline{u}-\bar{u} \leq \int_{\Omega} f(x, y)(\underline{u}(y, t)-\bar{u}(y, t)) d y \leq 0,
$$

in the case of $\int_{\Omega}(\underline{u}-\bar{u})_{+} d y=0$ in $\Omega$. Therefore, we obtain $\underline{u}(x, t) \leq \bar{u}(x, t)$ on $\partial \Omega \times(0, T)$, and $\underline{u}(x, t) \leq \bar{u}(x, t)$ in $\bar{\Omega} \times(0, T)$.

Next, we state the theorem of local existence and uniqueness without proof. 
Theorem (Local existence and uniqueness) Suppose that $p \geq 2, \alpha \geq 0, \beta>0, m>0$ and $k>0$, the nonnegative initial data $u_{0} \in L^{\infty}(\Omega) \cap W_{0}^{1, p}(\Omega)$ satisfies the compatibility conditions $u_{t}(x, 0)=\operatorname{div}\left(\left|\nabla u_{0}(x)\right|^{p-2} \nabla u_{0}(x)\right)-k u_{0}^{s}(x)$ for $x \in \Omega$ and $u_{0}(x)=\int_{\Omega} f(x, y) u_{0}(y) d y$ for $x \in \partial \Omega$. Then there exists a constant $T^{*}>0$ such that problem (1.5)-(1.7) admits a nonnegative solution $u \in C\left(0, T ; L^{\infty}(\Omega)\right) \cap L^{p}\left(0, T ; W_{0}^{1, p}(\Omega)\right)$ for each $T<T^{*}$. Furthermore, either $T^{*}=\infty$ or

$$
\lim _{t \rightarrow T^{*}} \sup \|u(x, t)\|_{\infty}=\infty
$$

Remark 1 The existence of local nonnegative solutions in time to problem (1.5)-(1.7) can be obtained by combining Theorem 1.2 in [4] with Theorem A4' in [19]. By the comparison principle above, we can get the uniqueness of the solutions to problem (1.5)-(1.7) with $\alpha+\beta \geq 1, m \geq 1$.

\section{Blow-up solutions and blow-up rate}

Comparing with the problem under a general homogeneous Dirichlet boundary condition, the existence of weight function in the boundary condition has a great influence on the global and non-global existence of solutions.

Theorem 1 Suppose that $\alpha+\beta>\max \{p-1, m\}$, then the solution of problem (1.5)-(1.7) blows up in finite time for arbitrary $f(x, y)>0$ and sufficiently large initial data.

Proof In order to prove the blow-up result, we need to establish a self-similar blow-up solution. Let

$$
\underline{u}(x, t)=\frac{\omega(r)}{v^{\xi}(r, t)}, \quad \text { with } 0 \leq r \leq R, \xi>1,0<t<3 T,
$$

where $\omega(r)=\cos ^{2}\left(\frac{\pi r}{2 R}\right), v(x, t)=4 T^{2}-\omega^{\delta}(t-T)^{2}=v_{1} v_{2}$, and

$$
v_{1}=2 T-\omega^{\frac{\delta}{2}}(t-T), \quad v_{2}=2 T+\omega^{\frac{\delta}{2}}(t-T) .
$$

It is obvious that $\underline{u}(r, t)$ blows up at $r=0$ as $t$ approaches $T$. Set

$$
\delta \geq 2, \quad T \leq \frac{1}{2} \quad \text { such that } v(r, t) \leq 1 .
$$

An explicit calculation yields

$$
\begin{aligned}
& r_{x_{i}}=\frac{x_{i}}{r}, \quad r_{x_{i} x_{i}}=-\frac{x_{i} x_{j}}{r^{3}}, \\
& \omega_{x_{i}}=\omega^{\prime} r_{x_{i}}=-\frac{\pi}{2 R} \sin \frac{\pi r}{R} r_{x_{i}}, \quad \nabla \omega=-\frac{\pi}{2 R} \sin \frac{\pi r}{R}, \\
& \omega_{x_{i} x_{j}}=\left(\omega^{\prime} r_{x_{i}}\right)_{x_{j}}=-\frac{\pi^{2}}{2 R^{2}} \cos \frac{\pi r}{R} \frac{x_{i} x_{j}}{r^{2}}-\frac{\pi}{2 R} \sin \frac{\pi r}{R}\left(-\frac{x_{i} x_{j}}{r^{3}}\right), \\
& -\triangle \omega=\frac{\pi}{2 R}\left(\frac{\pi}{R} \cos \frac{\pi r}{R}+\frac{n-1}{r} \sin \frac{\pi r}{R}\right), \\
& v_{x_{i}}=-\delta(t-T)^{2} \omega^{\delta-1} \omega^{\prime} r_{x_{i}}, \quad \nabla v=-\delta(t-T)^{2} \omega^{\delta-1} \nabla \omega, \quad v_{t}=-2 \omega^{\delta}(t-T),
\end{aligned}
$$




$$
\begin{aligned}
& \nabla \underline{u}=\frac{\nabla \omega v^{\xi}-\omega \xi \nu^{\xi-1} \nabla v}{v^{2 \xi}}=\frac{4 T^{2}+(\delta \xi-1) \omega^{\delta}(t-T)^{2}}{v^{\xi+1}}, \\
& \Delta \underline{u}=\nabla\left(\frac{\nu+\delta \xi \omega^{\delta}(t-T)^{2}}{v^{\xi+1}} \nabla \omega\right) \geq \frac{4 T^{2}+(\delta \xi-1) \omega^{\delta}(t-T)^{2}}{v^{\xi+1}} \Delta \omega .
\end{aligned}
$$

Then there exists $r_{0} \in(0, R)$ satisfying

$$
\begin{aligned}
& -\triangle \omega \leq 0, \quad r_{0} \leq r \leq R \\
& 0 \leq-\triangle \omega \leq \frac{n \pi^{2}}{2 R^{2}}, \quad \text { and } \quad \omega \geq \cos ^{2} \frac{\pi r_{0}}{2 R}, \quad 0 \leq r \leq r_{0}
\end{aligned}
$$

and

$$
\begin{aligned}
\frac{\partial \underline{u}}{\partial x_{i}}=\frac{\omega^{\prime} r_{x_{i}} v^{\xi}-\omega \xi v^{\xi-1} v_{x_{i}}}{v^{2 \xi}} & \frac{\partial^{2} \underline{u}}{\partial x_{i} \partial x_{j}}= \\
& \omega^{\prime \prime} v^{-\xi} r_{x_{i}} r_{x_{j}}+\xi \delta v^{-\xi-1}(t-T)^{2} \omega^{\delta-1}\left(\omega^{\prime}\right)^{2} r_{x_{i}} r_{x_{j}}+\omega^{\prime} v^{-\xi} r_{x_{i} x_{j}} \\
& +\xi \delta^{2} v^{-\xi-1}(t-T)^{2} \omega^{\delta-1}\left(\omega^{\prime}\right)^{2} r_{x_{i}} r_{x_{j}}+\xi \delta v^{-\xi-1}(t-T)^{2} \omega^{\delta} \omega^{\prime \prime} r_{x_{i}} r_{x_{j}} \\
& +\xi \delta^{2}(\xi+1) v^{-\xi-2}(t-T)^{2}(t-T)^{2} \omega^{2 \delta-1}\left(\omega^{\prime}\right)^{2} r_{x_{i}} r_{x_{j}} \\
& +\xi \delta v^{-\xi-1}(t-T)^{2} \omega^{\delta}\left(\omega^{\prime}\right) r_{x_{i} x_{j}} \\
= & v^{-\xi-1}\left(4 T^{2}+(\delta \xi-1) \omega^{\delta}(t-T)^{2} \omega^{\prime \prime} \frac{x_{i} x_{j}}{r^{2}}\right) \\
& +v^{-\xi-1}\left(4 T^{2}+(\delta \xi-1) \omega^{\delta}(t-T)^{2} \omega^{\prime}\left(-\frac{x_{i} x_{j}}{r^{3}}\right)\right) \\
& +\delta(\xi+1) v^{-\xi-2}(t-T)^{2} \omega^{\delta-1}\left(\omega^{\prime}\right)^{2}\left[4 T^{2}+(\delta \xi-1) \omega^{\delta}(t-T)^{2}\right] \frac{x_{i} x_{j}}{r^{2}} \\
& +\delta(\delta \xi-1) v^{-\xi-1}(t-T)^{2} \omega^{\delta-1}\left(\omega^{\prime}\right)^{2} \frac{x_{i} x_{j}}{r^{2}} .
\end{aligned}
$$

Therefore we have

$$
\frac{\partial^{2} \underline{u}}{\partial x_{i} \partial x_{j}} \geq v^{-\xi-1}(t-T)^{2} \omega^{\delta-1}\left(\omega^{\prime}\right)^{2}\left[4 T^{2}+(\delta \xi-1) \omega^{\delta}(t-T)^{2}\right] \Delta \omega \frac{x_{i} x_{j}}{r^{2}},
$$

and

$$
\sum_{j=1}^{N} \sum_{i=1}^{N} \frac{\partial \underline{u}}{\partial x_{i}} \frac{\partial^{2} \underline{u}}{\partial x_{i} \partial x_{j}} \frac{\partial \underline{u}}{\partial x_{j}} \geq v^{-\xi-1}(t-T)^{2} \omega^{\delta-1}\left(\omega^{\prime}\right)^{2}\left[4 T^{2}+(\delta \xi-1) \omega^{\delta}(t-T)^{2}\right] \triangle \omega|\nabla \omega|^{2} .
$$

In view of the above, this gives

$$
\begin{aligned}
\underline{u}_{t} & -\operatorname{div}\left(|\nabla \underline{u}|^{p-2} \nabla \underline{u}\right)-\underline{u}^{\alpha} \int_{0}^{t} \underline{u}^{\beta} d s+k \underline{u}^{m} \\
& =\underline{u}_{t}-\left(|\nabla \underline{u}|^{p-2} \triangle \underline{u}+(p-2)|\nabla \underline{u}|^{p-4}(\nabla \underline{u})^{T} H_{x}(\underline{u}) \cdot \nabla \underline{u}\right)-\underline{u}^{\alpha} \int_{0}^{t} \underline{u}^{\beta} d s+k \underline{u}^{m} \\
& =\underline{u}_{t}-\left(|\nabla \underline{u}|^{p-2} \triangle \underline{u}+(p-2)|\nabla \underline{u}|^{p-4} \sum_{j=1}^{N} \sum_{i=1}^{N} \frac{\partial \underline{u}}{\partial x_{i}} \frac{\partial^{2} \underline{u}}{\partial x_{i} \partial x_{j}} \frac{\partial \underline{u}}{\partial x_{j}}\right)-\underline{u}^{\alpha} \int_{0}^{t} \underline{u}^{\beta} d s+k \underline{u}^{m}
\end{aligned}
$$


Fang and Chang Boundary Value Problems 2014, 2014:8

Page 9 of 17

http://www.boundaryvalueproblems.com/content/2014/1/8

$$
\begin{aligned}
\leq & \frac{2 \xi \omega^{\delta+1}(t-T)}{v^{\xi+1}}-\left(\frac{4 T^{2}+(\delta \xi-1) \omega^{\delta}(t-T)^{2}}{v^{\xi+1}}\right)^{p-2}\left\{\frac{4 T^{2}+(\delta \xi-1) \omega^{\delta}(t-T)^{2}}{v^{\xi+1}}\right. \\
& \left.+(p-2) \frac{4 T^{2}+(\delta \xi-1) \omega^{\delta}(t-T)^{2}}{v^{\xi+1}}\right\} \Delta \omega|\nabla \omega|^{p-2}-\frac{\omega^{\alpha+\beta}}{v^{\xi \alpha}(r, t)} \int_{0}^{t} \frac{1}{v^{\xi \beta}(r, s)} d s+k \frac{\omega^{m}}{v^{m \xi}} \\
= & \frac{2 \xi \omega^{\delta+1}(t-T)}{v^{\xi+1}}-\frac{(p-1)\left[4 T^{2}+(\delta \xi-1) \omega^{\delta}(t-T)^{2}\right]^{p-1}}{v^{(\xi+1)(p-1)}} \Delta \omega|\nabla \omega|^{p-2} \\
& -\frac{\omega^{\alpha+\beta}}{v^{\xi \alpha}(r, t)} \int_{0}^{t} \frac{1}{v^{\xi \beta}(r, s)} d s+k \frac{\omega^{m}}{v^{m \xi}} .
\end{aligned}
$$

We will discuss the problem for two cases.

Case 1. $0<t \leq \frac{T}{2}, 3 T^{2}<v \leq 4 T^{2}$. We need to show that for sufficiently small $T$,

$$
\frac{2 \xi \omega^{\delta+1}(t-T)}{v^{\xi+1}}+k \frac{\omega^{m}}{v^{m \xi}}-\frac{(p-1)\left[4 T^{2}+(\delta \xi-1) \omega^{\delta}(t-T)^{2}\right]^{p-1}}{v^{(\xi+1)(p-1)}} \Delta \omega|\nabla \omega|^{p-2} \leq 0 .
$$

That is,

$$
\begin{aligned}
-\Delta \omega & \leq \frac{v^{(\xi+1)(p-2)-m \xi}\left[2 \xi \omega^{\delta+1}(T-t) v^{m \xi}-k \omega^{m} v^{\xi+1}\right]}{(p-1)\left[4 T^{2}+(\delta \xi-1) \omega^{\delta}(t-T)^{2}\right]^{p-1}|\nabla \omega|^{p-2}} \\
& \leq \frac{2 \xi \omega^{\delta+1}(T-t) v^{(\xi+1)(p-2)}}{(p-1)\left[4 T^{2}+(\delta \xi-1) \omega^{\delta}(t-T)^{2}\right]^{p-1}|\nabla \omega|^{p-2}} .
\end{aligned}
$$

Let $\delta$ be sufficiently large, satisfying $\xi \delta>2$. By the condition $3 T^{2}<v \leq 4 T^{2}, 0<\omega \leq 1$, and $|\nabla \omega|<\frac{\pi}{2 R}$, we just have to make the following equality hold:

$$
-\triangle \omega \leq \frac{1}{p-1} \frac{2 \xi \omega^{\delta+1}\left(3 T^{2}\right)^{(\xi+1)(p-2)}}{\left(3 \xi \delta T^{2}\right)^{p-1}} \frac{1}{\left(\frac{\pi}{2 R}\right)^{p-2}} .
$$

It is obvious that it holds for $r_{0}<r \leq R$.

For $0 \leq r<r_{0}$, choose $\xi>\frac{1}{p-2}, T<\min \left\{\frac{1}{2},\left[\frac{n \pi^{p}(\xi \delta)^{p-1}}{2^{p-1} 3^{\xi p-2 \xi-1} R^{p}}\left(\cos \frac{\pi r_{0}}{2 R}\right)^{-2(\delta+1)}\right]^{\frac{1}{2 \xi p-4 \xi-1}}\right\}$ such that

$$
\underline{u}_{t}-\operatorname{div}\left(|\nabla \underline{u}|^{p-2} \nabla \underline{u}\right)-\underline{u}^{\alpha} \int_{0}^{t} \underline{u}^{\beta} d s-k \underline{u}^{m} \leq 0 .
$$

Case 2. $\frac{T}{2}<t<3 T$, choose $\xi \beta>2$ and we can get

$$
\begin{aligned}
\underline{u}^{\alpha} \int_{0}^{t} \underline{u}^{\beta} d s & \geq \frac{\omega^{\alpha+\beta}}{v^{\xi \alpha} v_{2}^{\xi \beta}} \int_{0}^{t} \frac{1}{v_{1}^{\xi \beta}} d s=\frac{\omega^{\alpha+\beta}}{\omega^{\frac{\delta}{2}} v^{\xi \alpha} v_{2}^{\xi \beta}} \frac{1}{\beta \xi-1}\left(\frac{1}{v_{1}^{\xi \beta-1}}-\frac{1}{v_{1}^{\xi \beta-1}(0)}\right) \\
& =\frac{\omega^{\alpha+\beta-\frac{\delta}{2}}}{v^{\xi(\alpha+\beta)-1} v_{2}} \frac{1}{\beta \xi-1}-\frac{\omega^{\alpha+\beta-\frac{\delta}{2}}}{v^{\xi \alpha} v_{2}^{\xi \beta} v_{1}^{\xi \beta-1}(0)} \frac{1}{\beta \xi-1},
\end{aligned}
$$

and

$$
\begin{aligned}
\underline{u}_{t}- & \operatorname{div}\left(\mid \nabla \underline{u}^{p-2} \nabla \underline{u}\right)-\underline{u}^{\alpha} \int_{0}^{t} \underline{u}^{\beta} d s+k \underline{u}^{m} \\
\leq & \frac{4 \xi \omega^{\delta+1} T}{v^{\xi+1}}-\frac{(p-1)\left[4 T^{2}+(\delta \xi-1) \omega^{\delta}(t-T)^{2}\right]^{p-1}}{v^{(\xi+1)(p-1)}} \Delta \omega|\nabla \omega|^{p-2} \\
& -\frac{\omega^{\alpha+\beta-\frac{\delta}{2}}}{v^{\xi(\alpha+\beta)-1} v_{2}} \frac{1}{\beta \xi-1}+\frac{\omega^{\alpha+\beta-\frac{\delta}{2}}}{v^{\xi \alpha} v_{2}^{\xi \beta} v_{1}^{\xi \beta-1}(0)} \frac{1}{\beta \xi-1}+k \frac{\omega^{m}}{v^{m \xi}} .
\end{aligned}
$$


(i) $r_{0} \leq r \leq R$; we have $-\triangle \omega \leq 0$, then

$$
\begin{aligned}
\underline{u}_{t} & -\operatorname{div}\left(|\nabla \underline{u}|^{p-2} \nabla \underline{u}\right)-\underline{u}^{\alpha} \int_{0}^{t} \underline{u}^{\beta} d s+k \underline{u}^{m} \\
& \leq \frac{4 \xi \omega^{\delta+1} T}{v^{\xi+1}}-\frac{\omega^{\alpha+\beta-\frac{\delta}{2}}}{v^{\xi(\alpha+\beta)-1} v_{2}} \frac{1}{\beta \xi-1}+\frac{\omega^{\alpha+\beta-\frac{\delta}{2}}}{v^{\xi \alpha} v_{2}^{\xi \beta} v_{1}^{\xi \beta-1}(0)} \frac{1}{\beta \xi-1}+k \frac{\omega^{m}}{v^{m \xi}} .
\end{aligned}
$$

Since $\alpha+\beta>\max \{p-1, m\}$, choose $\xi \geq \max \left\{2(\alpha+\beta-1)^{-1}, 2 \beta^{-1},(\alpha+\beta-m)^{-1}\right\}$ and $\delta \geq$ $\max \{\alpha+\beta-1,2(\alpha+\beta-m), 2\}$ to satisfy $\xi(\alpha+\beta-p+1-m)>p$. However,

$$
\frac{v_{1}}{v_{1}(0)}=\frac{2 T-\omega^{\frac{\delta}{2}}(t-T)}{2 T+\omega^{\frac{\delta}{2}} T} \leq \frac{2 T-\omega^{\frac{\delta}{2}}\left(\frac{T}{2}-T\right)}{2 T+\omega^{\frac{\delta}{2}} T}=\frac{2+\frac{1}{2} \omega^{\frac{\delta}{2}}}{2+\omega^{\frac{\delta}{2}}}<1,
$$

thus we find

$$
\frac{v_{1}^{\xi \beta-1}}{v_{1}^{\xi \beta-1}(0)} \leq \frac{v_{1}}{v_{1}(0)} \leq \frac{2+\frac{1}{2} \omega^{\frac{\delta}{2}}}{2+\omega^{\frac{\delta}{2}}} \quad \text { for } \xi \beta-1>1
$$

Then

$$
\begin{aligned}
& \underline{u}_{t}-\operatorname{div}\left(|\nabla \underline{u}|^{p-2} \nabla \underline{u}\right)-\underline{u}^{\alpha} \int_{0}^{t} \underline{u}^{\beta} d s+k \underline{u}^{m} \\
& \leq \frac{\omega^{\alpha+\beta-\frac{\delta}{2}}}{v^{\xi(\alpha+\beta)-1} v_{2}} \frac{1}{\beta \xi-1}\left[4 \xi T(\xi \beta-1) v_{2} v^{\xi(\alpha+\beta-1)-2} \omega^{\frac{3 \delta}{2}-(\alpha+\beta-1)}\right. \\
& \left.+\frac{v_{1}^{\xi \beta-1}}{v_{1}^{\xi \beta-1}(0)}-1+k \frac{\omega^{m-\alpha-\beta+\frac{\delta}{2}} v_{2}}{v^{\xi(m-\alpha-\beta)+1}}(\xi \beta-1)\right] \\
& \leq \frac{\omega^{\alpha+\beta-\frac{\delta}{2}}}{v^{\xi(\alpha+\beta)-1} v_{2}} \frac{1}{\beta \xi-1}\left[8 \xi T(\xi \beta-1) \omega^{\frac{3 \delta}{2}-(\alpha+\beta-1)}\right. \\
& \left.+\frac{2+\frac{1}{2} \omega^{\frac{\delta}{2}}}{2+\omega^{\frac{\delta}{2}}}-1+4 T k(\xi \beta-1) \omega^{m-\alpha-\beta+\frac{\delta}{2}}\right]
\end{aligned}
$$

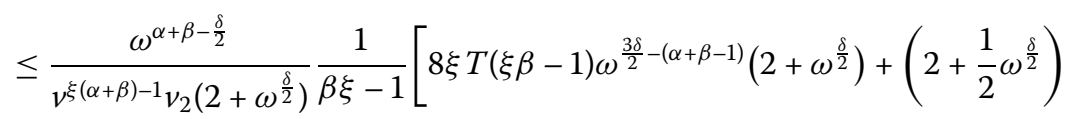

$$
\begin{aligned}
& \left.-\left(2+\omega^{\frac{\delta}{2}}\right)+4 k T\left(2+\omega^{\frac{\delta}{2}}\right)(\xi \beta-1) \omega^{m-\alpha-\beta+\frac{\delta}{2}}\right] \\
& \leq \frac{\omega^{\alpha+\beta-\frac{\delta}{2}}}{\nu^{\xi(\alpha+\beta)-1} v_{2}\left(2+\omega^{\frac{\delta}{2}}\right)} \frac{1}{\beta \xi-1}\left[24 \xi T(\xi \beta-1) \omega^{\frac{3 \delta}{2}-(\alpha+\beta-1)}\right. \\
& \left.-\frac{1}{2} \omega^{\frac{\delta}{2}}+12 k T(\xi \beta-1) \omega^{m-\alpha-\beta+\frac{\delta}{2}}\right]
\end{aligned}
$$

in the sense of $0<\omega \leq 1$ and $v_{2} \leq 4 T \leq 2$.

In order to get the result, we have to show that

$$
24 \xi T(\xi \beta-1) \omega^{\frac{3 \delta}{2}+(\alpha+\beta-1)}+12 k T(\xi \beta-1) \omega^{m-\alpha-\beta+\frac{\delta}{2}} \leq \frac{1}{2} \omega^{\frac{\delta}{2}} .
$$


Note that $0<\omega \leq 1$ and $\delta \geq \max \{\alpha+\beta-1,2(\alpha+\beta-m), 2\}$, we choose

$$
T \leq \min \left\{\frac{1}{2}, \frac{1}{24(2 \xi+k)(\xi \beta-1)}\right\}
$$

in which case we can get the result.

(ii) $0 \leq r<r_{0}$; we have $0 \leq \Delta \omega \leq \frac{n \pi^{2}}{2 R^{2}}$ and $\omega \geq \cos ^{2} \frac{\pi r_{0}}{2 R}$. Since $0<\omega \leq 1$ and $v(r, t) \leq 1$, we know that $\frac{4 \xi \omega^{\delta+1} T}{v^{\xi+1}} \leq \frac{4 \xi T}{v^{(\xi+1)(p-1)}}$, and

$$
\begin{aligned}
& \frac{(p-1)\left[4 T^{2}+(\delta \xi-1) \omega^{\delta}(t-T)^{2}\right]^{p-1}}{v^{(\xi+1)(p-1)}}(-\Delta \omega)|\nabla \omega|^{p-2} \\
& \leq \frac{(p-1)\left(4 \xi \delta T^{2}\right)^{p-1}}{v^{(\xi+1)(p-1)}} \frac{n \pi^{2}}{2 R^{2}}\left(\frac{\pi}{2 R}\right)^{p-2} \\
& =\frac{(p-1)\left(2 \xi \delta T^{2}\right)^{p-1}}{v^{(\xi+1)(p-1)}} \frac{n \pi^{p}}{R^{p}} .
\end{aligned}
$$

Let $A=\left(\cos ^{2} \frac{\pi r_{0}}{2 R}\right)^{\delta}, B=\frac{2+\frac{A}{2}}{2+A}$, it is obvious that $\frac{1}{2}<B<1$ and $\omega^{\delta} \geq A$. Substituting equation (3.2) into equation (3.1) gives

$$
\begin{aligned}
& \underline{u}_{t}-\operatorname{div}\left(|\nabla \underline{u}|^{p-2} \nabla \underline{u}\right)-\underline{u}^{\alpha} \int_{0}^{t} \underline{u}^{\beta} d s+k \underline{u}^{m} \\
& \leq \frac{4 \xi T}{v^{(\xi+1)(p-1)}}+\frac{(p-1)\left(2 \xi \delta T^{2}\right)^{p-1}}{v^{(\xi+1)(p-1)}} \frac{n \pi^{p}}{R^{p}}+\frac{\omega^{\alpha+\beta-\frac{\delta}{2}}}{v^{\xi \alpha} v_{2}^{\xi \beta} v_{1}^{\xi \beta-1}(0)} \frac{1}{\beta \xi-1} \\
& -\frac{\omega^{\alpha+\beta-\frac{\delta}{2}}}{v^{\xi(\alpha+\beta)-1} v_{2}} \frac{1}{\beta \xi-1}+k \frac{\omega^{m}}{v^{m \xi}} \\
& \leq \frac{4 \xi T+(p-1)\left(2 \xi \delta T^{2}\right)^{p-1} n \pi^{p}}{v^{(\xi+1)(p-1)} R^{p}}+\frac{\omega^{\alpha+\beta-\frac{\delta}{2}}}{v^{\xi \alpha} v_{2}^{\xi \beta} v_{1}^{\xi \beta-1}(0)} \frac{1}{\beta \xi-1}-\frac{\omega^{\alpha+\beta-\frac{\delta}{2}}}{v^{\xi(\alpha+\beta)-1} v_{2}} \frac{1}{\beta \xi-1}+\frac{k}{v^{m \xi}} \\
& =\frac{4 \xi T+(p-1)\left(2 \xi \delta T^{2}\right)^{p-1} n \pi^{p}+k R^{p}}{v^{(\xi+1)(p-1)+m \xi} R^{p}}+\frac{\omega^{\alpha+\beta-\frac{\delta}{2}}}{v^{\xi \alpha} v_{2}^{\xi \beta}} \frac{1}{\beta \xi-1}\left(\frac{1}{v_{1}^{\xi \beta-1}(0)}-\frac{B^{\xi \delta-1}}{v_{1}^{\xi \delta-1}}\right) \\
& -\frac{\left(1-B^{\xi \delta-1}\right) \omega^{\alpha+\beta-\frac{\delta}{2}}}{\nu^{\xi(\alpha+\beta)-1} v_{2}} \frac{1}{\beta \xi-1} \\
& \leq \frac{4 \xi T+(p-1)\left(2 \xi \delta T^{2}\right)^{p-1} n \pi^{p}+k R^{p}}{v^{(\xi+1)(p-1)+m \xi} R^{p}}-\frac{\left(1-B^{\xi \delta-1}\right) A^{\frac{\alpha+\beta}{\delta}-\frac{1}{2}}}{v^{\xi(\alpha+\beta)-1}} \frac{1}{\beta \xi-1} \\
& +\frac{\omega^{\alpha+\beta-\frac{\delta}{2}}}{v^{\xi \alpha} v_{2}^{\xi \beta}} \frac{1}{\beta \xi-1}\left(\frac{1}{v_{1}^{\xi \beta-1}(0)}-\frac{B^{\xi \delta-1}}{v_{1}^{\xi \delta-1}}\right)
\end{aligned}
$$

However, since $v_{1}=2 T-\omega^{\frac{\delta}{2}}(t-T) \leq 2 T+\frac{T}{2} \omega^{\frac{\delta}{2}}$ and $\frac{1}{2}<B<1$, it follows that

$$
\frac{1}{v_{1}^{\xi \beta-1}(0)} \leq \frac{B^{\xi \delta-1}}{v_{1}^{\xi \delta-1}}
$$

Finally, we need to show that

$$
\frac{4 \xi T+(p-1)\left(2 \xi \delta T^{2}\right)^{p-1} n \pi^{p}+k R^{p}}{v^{(\xi+1)(p-1)+m \xi} R^{p}} \leq \frac{\left(1-B^{\xi \delta-1}\right) A^{\frac{\alpha+\beta}{\delta}-\frac{1}{2}}}{\nu^{\xi(\alpha+\beta)-1}} \frac{1}{\beta \xi-1} .
$$


Because $T \leq \frac{1}{2}$ and $v \leq 4 T^{2} \leq 1$, we have to show

$$
\left[\frac{2 \xi+(p-1)(2 \xi \delta)^{p-1} n \pi^{p}+k R^{p}}{R^{p}}\right]\left(4 T^{2}\right)^{\xi(\alpha+\beta-p+1-m)-p} \leq \frac{\left(1-B^{\xi \delta-1}\right) A^{\frac{\alpha+\beta}{\delta}-\frac{1}{2}}}{\beta \xi-1} .
$$

In other words, we just need the following inequality:

$$
4 T^{2} \leq\left[\frac{\left(1-B^{\xi \delta-1}\right) A^{\frac{\alpha+\beta}{\delta}-\frac{1}{2}} R^{p}}{(\xi \beta-1)\left(4 \xi+(2 \xi \delta)^{p-1}\right) n \pi^{p}}\right]^{\frac{1}{\xi(\alpha+\beta-p+1-m)-p}}
$$

to hold. So choose $T$ to be small enough, and we can get

$$
\underline{u}_{t}-\operatorname{div}\left(|\nabla \underline{u}|^{p-2} \nabla \underline{u}\right)-\underline{u}^{\alpha} \int_{0}^{t} \underline{u}^{\beta} d s-k \underline{u}^{m} \leq 0
$$

For $x \in \partial \Omega, \omega(R)=0$,

$$
\underline{u}(R, t)=\frac{\omega(R)}{v^{\xi}(R, t)}=0 \leq \int_{\Omega} f(x, y) \underline{u} d y,
$$

choose $\underline{u}(r, 0)=\frac{\omega(r)}{v^{\xi}(r, 0)}=\frac{\omega(r)}{4 T^{2}-\omega^{\delta} T^{2}} \leq u_{0}$, then $\underline{u}(r, t)$ is the lower solution of problem (1.5)(1.7). This implies that the solution blows up in finite time for large enough initial data.

Theorem 2 Suppose that $\int_{\Omega} f(x, y) d y \geq 1$ for $x \in \partial \Omega$. If $\alpha+\beta>m \geq 1$, then the solution of problem (1.5)-(1.7) blows up in finite time for all strictly positive initial dates with $T$ sufficiently large.

Proof Consider the following problem:

$$
v^{\prime}(t)=T v^{\alpha+\beta}-k v^{m}, \quad v(0)=v_{0} .
$$

As $\alpha+\beta>m$, we know $v^{\alpha+\beta}+1>v^{m}$, and $T v^{\alpha+\beta}-k v^{m} \geq(T-k) v^{\alpha+\beta}-k$. Therefore, the solution of equation (3.3) is an upper solution of the following problem:

$$
v^{\prime}(t)=(T-k) v^{\alpha+\beta}-k, \quad v(0)=v_{0} .
$$

Since $T>k$ and $\alpha+\beta>1$, the solution of this problem blows up in finite time if $v_{0}>$ $\left(\frac{k}{T-k}\right)^{\frac{1}{\alpha+\beta}}$.

It is obvious that the solution of problem (3.3) is a lower solution of problem (1.5)-(1.7) when $\int_{\Omega} \varphi(x, y) d y \geq 1$ and $u_{0}(x)>v_{0}$. By Proposition $1, u(x, t)$ is a blow-up solution.

Suppose that the solution $u(x, t)$ of problem (1.5)-(1.7) with $p=2$ blows up in finite time, and let $U(t)=\max _{x \in \bar{\Omega}} u(x, t)$. We suppose that the initial data satisfies the following assumptions:

$\left(\mathrm{H}_{1}\right) \Delta u_{0}(x)+u_{0}^{\alpha} \int_{0}^{t} u_{0}^{\beta} d s-k u_{0}^{m}>0$.

$\left(\mathrm{H}_{2}\right)$ There exists a constant $\delta>0$ such that $\Delta u_{0}(x)+u_{0}^{\alpha} \int_{0}^{t} u_{0}^{\beta} d s-k u_{0}^{m}-\delta u_{0}^{\alpha+\beta} \geq 0$. 
Theorem 3 Suppose that $\int_{\Omega} f(x, y) d y \leq 1$ for $x \in \partial \Omega$. If $\alpha+\beta>1, u_{0}(x)$ satisfies condition $\left(\mathrm{H}_{1}\right)-\left(\mathrm{H}_{2}\right)$, and $u(x, t)$ is the blow-up solution of problem (1.5)-(1.7) in finite time $T$ with $p=2$, then the blow-up rate is

$$
c(T-t)^{-\frac{1}{\alpha+\beta-1}} \leq u(x, t) \leq C(T-t)^{-\frac{1}{\alpha+\beta-1}},
$$

where $c=[(\alpha+\beta-1) T]^{-\frac{1}{\alpha+\beta-1}}$ and $C=[\delta(\alpha+\beta-1)]^{-\frac{1}{\alpha+\beta-1}}$.

Remark 2 Choose $\Omega=(1,2), \alpha=2, \beta=1, m=2$ and $f(x, y)=\frac{1}{8} x y$, one can easily verify that $u_{0}(x)=x^{2}$ satisfies $\left(C_{1}\right)-\left(C_{2}\right)$, conditions in Theorem 3 are thus valid.

Lemma 1 If $u_{0}(x)$ satisfies condition $\left(\mathrm{H}_{1}\right)-\left(\mathrm{H}_{2}\right), p=2$, then there exists a positive constant $c=[(\alpha+\beta-1) T]^{-\frac{1}{\alpha+\beta-1}}$ such that $U(t) \geq c(T-t)^{-\frac{1}{\alpha+\beta-1}}$.

Proof It is obvious that $U(t)$ is Lipschitz continuous and differentiable almost everywhere. By equation (1.5) with $p=2$ and $\Delta U(t) \leq 0$, it yields

$$
U^{\prime}(t) \leq U^{\alpha} \int_{0}^{t} U^{\beta} d s-k U^{m} \leq T U^{\alpha+\beta}
$$

and thus

$$
-\left(U^{1-\alpha-\beta}(t)\right)^{\prime} \leq T(\alpha+\beta-1) .
$$

Integrating the result above over $(t, T)$, we can obtain the conclusion.

Proof of Theorem 3 Let $J=u_{t}-\delta u^{\alpha+\beta}$, where $\delta>0$ is sufficiently small. Since $\alpha+\beta>1$, we have

$$
\begin{aligned}
& J_{t}= u_{t t}-\delta(\alpha+\beta) u^{\alpha+\beta-1} u_{t} \\
&= \Delta u_{t}+\alpha u^{\alpha-1} u_{t} \int_{0}^{t} u^{\beta} d s+u^{\alpha+\beta}-k m u^{m-1} u_{t}-\delta(\alpha+\beta) u^{\alpha+\beta-1} u_{t}, \\
& \begin{aligned}
\triangle I= & \Delta u_{t}-\delta(\alpha+\beta) u^{\alpha+\beta-1} u_{t}+\delta(\alpha+\beta) u^{2 \alpha+\beta-1} \\
\quad & \quad \int_{0}^{t} u^{\beta} d s-\delta(\alpha+\beta)(\alpha+\beta-1) u^{\alpha+\beta-2}|\nabla u|^{2}, \\
J_{t}-\Delta J \geq & \alpha u^{\alpha-1} u_{t} \int_{0}^{t} u^{\beta} d s+u^{\alpha+\beta}-\delta(\alpha+\beta) u^{2 \alpha+\beta-1} \int_{0}^{t} u^{\beta} d s-k m u^{m-1} u_{t} \\
= & \left(\alpha u^{\alpha-1} \int_{0}^{t} u^{\beta} d s-k m u^{m-1}\right) J+u^{\alpha+\beta}\left(1-\delta \beta u^{\alpha-1} \int_{0}^{t} u^{\beta} d s+k m u^{m-1} \delta\right),
\end{aligned}
\end{aligned}
$$

so we can choose $\delta>0$ to be small enough and thus obtain

$$
J_{t}-\triangle J \geq \alpha u^{\alpha-1} J \int_{0}^{t} u^{\beta} d s
$$


On the other hand, as $(x, t) \in \partial \Omega \times(0, T)$, we get

$$
\begin{aligned}
J & =u_{t}-\delta u^{\alpha+\beta}=\int_{\Omega} f(x, y) u_{t} d y-\delta\left(\int_{\Omega} f(x, y) u d y\right)^{\alpha+\beta} \\
& =\int_{\Omega} f(x, y) J d y+\delta \int_{\Omega} f(x, y) u^{\alpha+\beta} d y-\delta\left(\int_{\Omega} f(x, y) u d y\right)^{\alpha+\beta} .
\end{aligned}
$$

Let $0<F(x)=\int_{\Omega} f(x, y) d y \leq 1$, By Jensen's inequality, this gives

$$
\begin{aligned}
J & \geq \int_{\Omega} f(x, y) J d y+\delta F(x)\left[\int_{\Omega} f(x, y) u \frac{d y}{F(x)}\right]^{\alpha+\beta}-\delta\left(\int_{\Omega} f(x, y) u d y\right)^{\alpha+\beta} \\
& \geq \int_{\Omega} f(x, y) J d y
\end{aligned}
$$

and

$$
J(x, t)=u_{t}-\delta u^{\alpha+\beta}=\Delta u+u^{\alpha} \int_{0}^{t} u^{\beta} d s-\delta u^{\alpha+\beta} .
$$

It follows from the assumptions of $\left(\mathrm{H}_{1}\right)-\left(\mathrm{H}_{2}\right)$ that $J(x, 0) \geq 0$. Therefore, it is easy to deduce that $J(x, t) \geq 0$ for $(x, t) \in \bar{\Omega} \times[0, T)$. That is, $u_{t} \geq \delta u^{\alpha+\beta}$ and integrating this over $(t, T)$ yields $u \leq C(T-t)^{-\frac{1}{\alpha+\beta-1}}$. Combining the results with Lemma 1 , we obtain the desired result.

\section{Global existence of solutions}

In this section, we give sufficient conditions of the global existence of solutions.

Theorem 4 Suppose that $\int_{\Omega} f(x, y) d y<1$ for $x \in \partial \Omega$. If $\alpha+\beta \leq \max \{p-1, m\}$, then the solution of problem (1.5)-(1.7) exists globally for small initial data.

Proof Let $\bar{u}(x, t)=q e^{n t} \psi(x)$, where $q, n>0$ are determined later, and $\psi(x)$ solves the following problem:

$$
\begin{aligned}
& -\operatorname{div}|\nabla \psi|^{p-2} \nabla \psi=1, \quad x \in \Omega, \\
& \psi(x)=\int_{\Omega} f(x, y) d y, \quad x \in \partial \Omega,
\end{aligned}
$$

where $0<\psi(x)<1$. Let $\eta_{1}=\max _{\bar{\Omega}} \psi(x), \eta_{2}=\max _{\bar{\Omega}} \psi(x)$,

$$
\begin{aligned}
\bar{u}_{t} & -\operatorname{div}\left(|\nabla \bar{u}|^{p-2} \nabla \bar{u}\right)-\bar{u}^{\alpha} \int_{0}^{t} \bar{u}^{\beta} d s+k \bar{u}^{m} \\
& =q n e^{n t} \psi+q^{p-1} e^{n(p-1) t}-\frac{1}{n \beta} q^{\alpha+\beta} e^{n(\alpha+\beta) t} \psi^{\alpha+\beta}+k q^{m} e^{m n t} \psi^{m} \\
& \geq q^{p-1} e^{n(p-1) t}-\frac{1}{n \beta} q^{\alpha+\beta} e^{n(\alpha+\beta) t} \psi^{\alpha+\beta}+k q^{m} e^{m n t} \psi^{m}
\end{aligned}
$$

since $\alpha+\beta<\max \{p-1, m\}$, choosing

$$
n>\max \left\{\frac{1}{k \beta}\left(q \eta_{1}\right)^{\alpha+\beta-m}, \frac{1}{\beta} q^{\alpha+\beta-p+1} \eta_{2}^{\alpha+\beta}\right\},
$$


we can infer that

$$
\bar{u}_{t}-\operatorname{div}\left(|\nabla \bar{u}|^{p-2} \nabla \bar{u}\right)-\bar{u}^{\alpha} \int_{0}^{t} \bar{u}^{\beta} d s+k \bar{u}^{m} \geq 0
$$

For $x \in \partial \Omega, 0<\psi(x)<1$

$$
\bar{u}(x, t)=q e^{n t} \int_{\Omega} f(x, y) d y \geq \int_{\Omega} f(x, y) \bar{u} d y .
$$

Selecting $u_{0}(x)<q \eta_{1}$, we can deduce that the result holds.

Theorem 5 Suppose that $\int_{\Omega} f(x, y) d y \leq \rho<1$ for $x \in \partial \Omega$. If $p-1<\min \{\alpha, \beta+1, m\}$, and $\alpha+\beta>m$, then the solution of problem (1.5)-(1.7) exists globally for small initial data.

Proof Suppose that $\eta(x)$ solves the following problem:

$$
\begin{aligned}
& -\operatorname{div}|\nabla \eta|^{p-2} \nabla \eta=1, \quad x \in \Omega, \\
& \eta(x)=0, \quad x \in \partial \Omega,
\end{aligned}
$$

in which $0<\eta(x)<1$. Let $C=\max _{\bar{\Omega}} \eta(x)$.

Set $\bar{u}(x, t)=k_{1}(A+t)^{-r}\left(\frac{\rho}{1-\rho}+\frac{\eta(x)}{C}\right)$, where $A, k_{1} \geq 1$ and $0 \leq r \leq \frac{1}{p-2}$, then

$$
\bar{u}_{t}=-k_{1} r(A+t)^{-r-1}\left(\frac{\rho}{1-\rho}+\frac{\eta(x)}{C}\right) \geq-k_{1} r(A+t)^{-r-1} \frac{1}{1-\rho} \geq-k_{1} r(A+t)^{-r(p-1)} \frac{1}{1-\rho},
$$

and

$$
\begin{aligned}
-\bar{u}^{\alpha} \int_{0}^{t} \bar{u}^{\beta} d s & =-k_{1}^{\alpha+\beta}(A+t)^{-r \alpha}\left(\frac{\eta(x)}{C}+\frac{\rho}{1-\rho}\right)^{\alpha+\beta} \int_{0}^{t}(A+t)^{-r \beta} d s \\
& \geq-\frac{k_{1}^{\alpha+\beta}}{1-r \beta}(A+t)^{1-r \alpha-r \beta}\left(\frac{\eta(x)}{C}+\frac{\rho}{1-\rho}\right)^{\alpha+\beta} .
\end{aligned}
$$

For $p-1<\min \{\alpha, \beta+1, m\}$, we choose $0 \leq r \leq \frac{1}{p-2}$ such that $1-r(\alpha+\beta-m)<0$ and $r \beta \leq 1$. It follows that

$$
-\bar{u}^{\alpha} \int_{0}^{t} \bar{u}^{\beta} d s \geq-\frac{k_{1}^{\alpha+\beta}}{1-r \beta}(A+t)^{1-r \alpha-r \beta}\left(\frac{1}{1-\rho}\right)^{\alpha+\beta},
$$

and

$$
\begin{aligned}
\bar{u}_{t}- & \operatorname{div}\left(|\nabla \bar{u}|^{p-2} \nabla \bar{u}\right)-\bar{u}^{\alpha} \int_{0}^{t} \bar{u}^{\beta} d s+k \bar{u}^{m} \\
\geq & -k_{1} r(A+t)^{-r(p-1)} \frac{1}{1-\rho}+k_{1}^{p-1}(A+t)^{-r(p-1)}-\frac{k_{1}^{\alpha+\beta}}{1-r \beta}(A+t)^{1-r \alpha-r \beta}\left(\frac{1}{1-\rho}\right)^{\alpha+\beta} \\
& +k k_{1}^{m}(A+t)^{-m r}\left(\frac{\eta(x)}{C}+\frac{\rho}{1-\rho}\right)^{m} \\
= & k_{1}(A+t)^{-r(p-1)}\left(\frac{-r}{1-\rho}+\frac{k_{1}^{p-2}}{2}\right)+k_{1}^{p-1}(A+t)^{-r(p-1)}
\end{aligned}
$$




$$
\begin{aligned}
& \times\left[\frac{1}{2}-\frac{k_{1}^{\alpha+\beta-p+1}}{1-r \beta}(A+t)^{1-r(\alpha+\beta-p+1)}\left(\frac{1}{1-\rho}\right)^{\alpha+\beta}\right. \\
& \left.+k k_{1}^{m-p+1}(A+t)^{-r(m-p+1)}\left(\frac{\eta(x)}{C}+\frac{\rho}{1-\rho}\right)^{m}\right] .
\end{aligned}
$$

Since $A, k_{1} \geq 1$, choosing

$$
k_{1} \geq \max \left\{\left(\frac{2 r}{1-\rho}\right)^{p-2}, 1\right\}
$$

and

$$
A \geq \max \left\{\left[k(1-r \beta)\left(\frac{1-\rho}{k_{1}}\right)^{\alpha+\beta-m}\right]^{\frac{1}{1-r(\alpha+\beta-m)}}, 1\right\}
$$

we can get

$$
\bar{u}_{t}-\operatorname{div}\left(|\nabla \bar{u}|^{p-2} \nabla \bar{u}\right)-\bar{u}^{\alpha} \int_{0}^{t} \bar{u}^{\beta} d s+k \bar{u}^{m} \geq 0 .
$$

On the other hand, for $x \in \partial \Omega$ and sufficiently large $A$, we have

$$
\begin{aligned}
\bar{u}(x, t) & =k(A+t)^{-r} \frac{\rho}{1-\rho} \geq k(A+t)^{-r} \frac{1}{1-\rho} \int_{\Omega} f(x, y) d y \\
& \geq \int_{\Omega} k(A+t)^{-r}\left(\frac{\eta(x)}{C}+\frac{\rho}{1-\rho}\right) f(x, y) d y \\
& =\int_{\Omega} \bar{u} f(x, y) d y .
\end{aligned}
$$

Choosing $u_{0}$ to be sufficiently small such that $u_{0} \leq k A^{-r}\left(\frac{\eta(x)}{C}+\frac{\rho}{1-\rho}\right)$, we can conclude that $\bar{u}$ is an upper solution of problem (1.5)-(1.7). The proof is completed.

Theorem 6 Suppose that $\int_{\Omega} f(x, y) d y<1$ for $x \in \partial \Omega$. If $\alpha+\beta>m$, then the solution of problem (1.5)-(1.7) exists globally for small enough initial data.

Proof Choosing $\bar{u}(x, t)=A$ and $A>\max \left\{\left(\frac{k}{T}\right)^{\frac{1}{\alpha+\beta-1}}, u_{0}(x)\right\}$, it is easy to see that the result holds.

Competing interests

The authors declare that they have no competing interests.

Authors' contributions

All authors contributed equally to the manuscript and read and approved the final manuscript.

\section{Acknowledgements}

This work is supported by the Natural Science Foundation of Shandong Province of China (ZR2012AM018) and the Fundamental Research Funds for the Central Universities (No. 201362032). The authors would like to warmly thank all the reviewers for their insightful and constructive comments. 


\section{References}

1. Friedman, A: Monotonic decay of solutions of parabolic equations with nonlocal boundary conditions. Q. Appl. Math 44(3), 401-407 (1986)

2. Pao, CV: Nonlinear Parabolic and Elliptic Equations. Plenum, New York (1992)

3. Akila, Y: On a nonlocal parabolic problem. Demonstr. Math. 42(4), 745-755 (2009)

4. Wang, YL, Mu, CL, Xiang, ZY: Blow-up of solutions to a porous medium equation with nonlocal boundary condition. Appl. Math. Comput. 192, 579-585 (2007)

5. Li, YX, Xie, CH: Blow-up for semi-linear parabolic equations with nonlinear memory. Z. Angew. Math. Phys. 55, 15-27 (2004)

6. Souplet, P: Monotonicity of solutions and blow-up for semilinear parabolic equations with nonlinear memory. Z. Angew. Math. Phys. 55, 28-31 (2004)

7. Zhou, J, Mu, CL, Lu, F: Blow-up and global existence to a degenerate reaction-diffusion equation with nonlinear memory. J. Math. Anal. Appl. 333, 1138-1152 (2007)

8. Du, LL, Mu, CL: Global existence and blow-up analysis to a degenerate reaction-diffusion system with nonlinear memory. Nonlinear Anal., Real World Appl. 9, 303-315 (2008)

9. Zhou, J, Mu, CL, Fan, MS: Global existence and blow-up to a degenerate reaction-diffusion system with nonlinear memory. Nonlinear Anal., Real World Appl. 9, 1518-1534 (2008)

10. Liu, DM, Mu, CL: Blow-up analysis for a semi-linear parabolic equation with nonlinear memory and nonlocal nonlinear boundary condition. Electron. J. Qual. Theory Differ. Equ. 51, 1-17 (2010)

11. Bebernes, J, Eberly, D: Mathematical Problems from Combustion Theory. Springer, New York (1989)

12. Furter, J, Grinfield, M: Local vs. non-local interactions in populations dynamics. J. Math. Biol. 27, $65-80$ (1989)

13. Calsina, A, Perello, C, Saldana, J: Non-local reaction-diffusion equations modelling predator-prey co-evolution. Publ. Mat. 32, 315-325 (1994)

14. Allegretto, W, Fragnelli, G, Nistri, P, Papin, D: Coexistence and optimal control problems for a degenerate predator-prey model. J. Math. Anal. Appl. 378, 528-540 (2011)

15. Kastenberg, WE: Space dependent reactor kinetics with positive feed-back. Nukleonika 11, 126-130 (1968)

16. Pao, CV: Asymptotic behavior of solutions of reaction-diffusion equations with nonlocal boundary conditions. J. Comput. Appl. Math. 88(1), 225-238 (1998)

17. Pao, CV: Numerical solutions of reaction-diffusion equations with nonlocal boundary conditions. J. Comput. Appl. Math. 136(1-2), 227-243 (2001)

18. Diaz, Jl: Nonlinear Partial Differential Equations and Free Boundaries: Elliptic Equations. Vol. I. Pitman, London (1985)

19. Soupllet, P: Blow-up in nonlocal reaction-diffusion equations. SIAM J. Math. Anal. 29(6), 1301-1334 (1998)

10.1186/1687-2770-2014-8

Cite this article as: Fang and Zhang: Global existence and blow-up of solutions for $p$-Laplacian evolution equation with nonlinear memory term and nonlocal boundary condition. Boundary Value Problems 2014, 2014:8

\section{Submit your manuscript to a SpringerOpen ${ }^{\circ}$ journal and benefit from:}

- Convenient online submission

- Rigorous peer review

Immediate publication on acceptance

Open access: articles freely available online

- High visibility within the field

- Retaining the copyright to your article 